\section{CONTRIBUIÇAO AO ESTUDO DA VASCULARIZAÇĀO ARTERIAL DO TIMO EM FETOS DE BOVINOS DE ORIGEM EUROPÉIA (BOS TAURUS)}

PEDRO PRIMO BOMBONATO Professor Assistente Doutor Faculdade de Medicina Veterinaria Zootecnia da USP

VICENTE BDRELLI Professor Titular Faculdade de Medicina Veterinaria e Zootecnia da USP

ANTONIO FERNANDES FILHO Professor Rdjunto Faculdade de Medicina Veterinaria Zootecnnia da USP
BOMBONATO, P.P.; BORELLI, U., FERNANDES FILHO, A. Contribuicyo ao estudo do vescularizacto arta rial do timo en fetos de bovinos de origen europela (Bos tourus). Rev.Fac. Med.vet.Zootec. Univ.5.Poulo, 23(1):31.38, 1986.

RESUMO: Foi estudada orlgem, o numero a ordenacso das arterias IImicas en 30 fetos de bovinos de origem europlid (Bos tourus), sendo 16 remes 14 machos em diferentes estagios de desenvolvimento, tendo sido injetsos com solucso de Neoprene latex $450^{\circ}$ e flxados em solucso aquos de farmol a 10,08. Os resultados Indican que o tlmo pecebe contribulcoles procedentes do tronco braquiocef lilico. das pas carotidas comuns difeita esquerda, da $A$. toracica interna esquerda. da A. Torbcica externa esquerda da A. tiroidea cranial esquerda. Independentemente das suas origens, os colaterais tielcos estyo presente em numero de olto - 8 vezes, sete - 7 vezes. seis - 6 vezes, cinco-4 vezes, dez - 3 vezes, nove i vez. cabendo sollentar que quonto ordenacso, cada preparasjo possul arranjo vascular proprio.

UNITERMO5: Anstomia, Bovinos: Arteriasi Timo

\section{INTRODUCAO E LITERATURA}

No que tange a estudos relativos vascularizaclo arterial, podemos afirmar que o timo dos animais domésticos tem sido pouco explorado. Neste sentido procuramos analisar em fetos de bovinos de origem europeia (Bos taurus); a origem, o numero e a ordenacao das arterias destinadas ao timo, buscando, assim, methor conhecer a irrigacto de tyo importante brgyo que, sem duvida, representa um dos componentes de major importe complexo imunitbrio.

Nos compendios de anatomia veterintria verificamos que pouca atenço e dada anatomia do timo e en especial, a seu aporte sangulneo, of erecendo apenas dados genericos e por vezes imprecisos. Dentre os autores que ocupam da anatomia do timo dos bovinos, encontramos MARTIN, 7 (1902), MARTIN \& SCHAUDER 8 (1938) E ELLENBERGER \& BAUM, 3 (1977) atendo-se apenas as origens dos vasos timicos, onde destacam a participacyo das Aa. carbtidas comuns, subelavia toracica interna. KOCH, 5 (1963), fazendo o mesmo tlpo de relato, acrescenta a contribuicro que o tronco braquiocefblico oferece, enquanto GETTY, 4 (1975) esclarece que os colaterais enderecados ao timo syo procedentes das $A$ a. carotidas comuns, subclbuia e torbelca interna esquerda. Dos trabalhos especiallzados, destacamos a pesquisa de DENIZ, 2 (1964) que, estudando as arterias e veias maiores do timo, mediante injecyo de latex, en fetos de bouinos, cujo comprimento do parietal ao coccix variava de 18 a $82 \mathrm{~cm}$, apresenta os seguintes resultados: o numero de arterias destinadas a timo cervical estaria na dependencia do tamanho do feto; encontrando-se tres - 15 casos), quatro- (10 casos), cinco- ( casos) e seis - $(2$ casos) Aa. thymicae cervicales sinn.. oriundas indiretamente da A. carotis communis e ainda uma - ( 1 caso), tres - (10 casos), quatro- $(5$ casos) e cinco - ( 4 casos) Aa. thymicae cervicales dextrae, procedente indiretamente da $A$. carotis communis. Acrescenta, ainda, a autor que o tpice cranial do hilo nutrido por un ramo lateral da $A$.' thyreoidea e que existem tres afluentes arteriais na area do timo peitoral, isto e, a A. thymica thoracica, presente em duplicidade 7 vezes. oriunda da borda dorsolateral do truncus brachiocephalicus communis; o ramus thyml. cus thoracicus da $A$. vertebralis, que deixou de aparecer en 12 casos.

LUCKHAUS, 6 (1966), baseando-se na descricyo de DENIZ, 2, estudou 27 fetos de bovinos, cujos comprimentos variavam de 17 a $80 \mathrm{~cm}$, concluindo que a pars cranialis thymi nutrida por numero variavel de arterias procedentes do tronco carotideo, denominada de $A$ a. thymicae craniales propriae os ramos diretos e de Aa. thymicae craniales accessoriae os ramos indiretos, sendo estas freqoentemente derivadas da $A$. thyraidea cranialis. O autor relata, ainda. 
a presenca de uma arteria tlmica nascendo do ramus descendens da $A$. occipitalis, afirmando tamben que a massa timica subbasilaris mostrava-se irrigada por ramos da A. palatina ascendens, em todos os casos. OLIVEIRA et alíl, 9 (1979), ao estudarem a ramescencia da a. tiroidea cranial em fetos de bovinos mesticos azebuados, sendo 29 femeas e 13 machos, e de comprimento varlando de 30 a $80 \mathrm{~cm}$, af irmam que para o timo: partem desta arteria contribuicbes, bilateralmente, en uma femea $(2,38 \%)$, unilateralmente, pelo lado direjto, en 7 machos $(16,66 x)$ e 5 feneas $(11,908)$ e, delo lado esquerdo, em dois machos (4,768) e duas femeas $(4,76 x)$.

BOMBONATO, 1 (1982), analisando a vascularizacso arterial do timo em 30 fetos de bovinos azebuados machos e femeas, cujo comprimento da crista nucal ao coccix oscilava entre 30 e $85 \mathrm{~cm}$, chegou is seguintes conclusies: 1) o timo nutrido por colaterais procedentes do tronco braquiocefalico comum, da $A$. toracica interna esquerda, do tronco omocervical, das Ra. carbtidas comuns direita e esquerda, da A. tiroidea cranial, em numero de nove - 10 vezes $(33,38 \pm 8,6)$, sete - 6 vezes $(20,08 \pm$ $7,3)$, oito 4 - vezes $(13,35 \pm 6,2)$, einco 3 vezes $(10,05 \pm 5,5)$, seis -3 vezes $(10,08 \pm 5,5)$, dez -2 vezes $(6,78 \pm 4,5)$, quatro -1 vez $(3,35 \pm 3,3)$, onze - 1 vez $(3,38 \div 3,3) ; 2)$ a poreyo torbcica do timo e irrigada por colaterais orjundos exclusivamente do tronco braquiocefalico comum 15 vezes $(50,0 \%+9,1)$, ou deste associado a contribuiczes da $A$. toracica interna esquerda 19 vezes $(36,79 \pm 8,8)$ ou do tronco omocervical 4 vezes $(13,3 x \pm 6,2)$; 3$)$ os colaterais destinados a porešo torbcica do timo, independentemente de sua origem,

mostram-se em numero de um a quatro, mais precisamente dois - 13 vezes $(43,38 \pm 9,1)$, tres - 12 vezes $(40,08 \pm 8,9)$, un - 3 vezes $(10,08 \pm 5,5)$ e quatro - 2 vezes (6,78 \pm $4,5) ; 4)^{-}$porcyo cervical do timo e servida apenas por colaterais originados de ambas as $A$ a. carbtidas comuns, 9 vezes $(30,08 \pm 8,4)$ ou simplesmente, da $A$, cardtida comum direita, 1 vez $(3,38+3,3)$ ou alinda de contribuicto delas procedentes, associadas a colaterais da $A$. tiroidea cranial, 9 vezes $(30,08 \pm 8,4)$, do tronco omocervical, 4 vezes $(13,38 \pm 6,2)$, da $A$. tortcica interna esquerda, 4 vezes $(13,38 \pm$ $6,2)$, e do tronco omocervical, juntamente com da $A$. tiroidea cranial, 3 vezes $(10,08 \pm 5,5) ; 5)$ os colaterais enderefados a porcyo cervical do timo, independentemente de sua origem, apresentam-sa em numero de dois a nove, mais exatamente seis - 7 vezes $(23,38 \pm 7,7)$, sete -7 vezes $(23,38$ $\pm 7,7)$, quatro - 6 vezes $(20,0 \% \pm 7,3)$, tres - 3 vezes $(10,0 \% \pm 5,5)$, cinco- 3 vezes $(10,0 \% \pm 5,5)$, dois - 2 vezes $(6,7 \%$. $4,5)$, oito -1 vez $(3,3 \% \pm 3,3)$, nove - 1 vez $(3,3 \% \pm 3,3) ; 6)$ diférencas estatisticamente significantes, Pelativamente ao sexo, no que tange d nutricao do tlmo, foram identificadas apenas quando da participasyo de colaterais oriundos da $A$. torbcica esquerda e dos ramos que, indiretamente, a $A$. carotida comum esquerda cede a glandula; 7$)$ considerados, conjuntamente, o numero, procedencia e ordenacro das arte$r$ ias destinadas ao timo identificam modalidades de vascularizacro proppia de cada casn

\section{MATERIAL E METODD}

Valemo-nos do estudo de 30 fetos de bovinos de origem europtia (Bos taurus), 16 femeas e 14 machos, em diferentes estagios de desenvolvimento e apresentando comprimento varibuel de 24 a $82 \mathrm{~cm}$, dimensyo esta considerada da crista nucal darticulacyo entre as duas prineiras vertebras coccigenas. Estes animais foram obtidos em Matadouros no Municlpio de Pelotas, R.S.

Apos a coleta, procedemos ao preenchimento das arterias destinadas ao timo, mediante injecro da $A$. aorta com solucro de Neoprene latex "45n" tingida por corante especificon. As pecas assim preparadas e depolis de fixadas en solucyo aquosa de formol a 10,08, foram submetidas disseccyo das arterias enderecadas ao $t 1$ mo, tanto para a pars thorbcica (poreyo torbcica) de forma aproximadamente trapezolde, como tambem para a pars cervicalis (poreyo cervical), de configuraclo semelhante a um ipsilon; divisyo esta adotada pela maioria dos autores e que tem como reparo a margem cranial do primeiro par de costelas. Para andllse descricyo dos resultados, confeccionamos desenhos esquematicos, destacando - ponto de penetraço dos vasos no orglo.

Os resultados obtidos foram estudados estatlsticamente, com auxilio do teste $x^{2}$, para $a=5,0 \%$, ainda tratados pela regressyo linear e testada sua correlaclo, com grau de liberdade 28 e sequranca de $95,0 \%$.

\section{RESULTADOS}

- estudo por nos realizado apresentou os resultados que passamos a descrever, obedecendo divisgo do timo em duas porcoles, a torbcica a cervical.

Assim, a porclo torbcica e nutrida parcialmente 20 vezes $(66,68 \div 8,6)$ por colaterais procedentes do tronco braquiocefalleo, associados 18 vezes $(60,08 \pm 8,9)$ a colaterais oriundos da $A$. toracica interna esquerda e 2 vezes $(6,64 \pm 4,5)$ a colaterais da $A$. torbcica externa esquerda. Nestes casos o tronco braquiocefalico a A. torácjca interna esquerda cedem colaterais em numero, respectivamente, de um e um - 13 vezes, ou dois e un 5 - vezes, enquanto que

* Du Pont do Brasil S.A. 
- tronco braquiocef́lico e a. toracica externa esquerda fornecem, por ordem, uma e uma - 1 vez duas e uma - 1 vez contribuifores.

Nas outras preparactos, 10 vezes $(33,38 \div 8,6)$, esta mesma parte do drgao acha-se irrigada exclusivamente por colaterais originados do tronco braquiocefalico, isto e, mais comumente por dois vasos - 7 vezes ou apenas un - 3 vezes.

Independentemente de sua origem, os vasos timicos ora descritos atingem unicamente a porcro toracica do orgyo, excecro feita a 2 casos em que ramos da $A$. toracica externa esquerda dividem-se para alcancar tamben a porczo cervical do timo.

No que diz respeito porcyo cervical do tlmo, esta acha-se nutrida mais frequentemente, 21 vezes $(70,08 \div 8,4)$, por colaterais oriundos das Aa. carotidas comuns direjta e esquerda que, considerados em relaço ao seu numero total e ao seu lado de origem apresentam, respectivamente, a seguinte distribuicso: seis -7 vezes, sendo tres e tres - 4 vezes, dois equatro - 2 vezes e quatro e dois - 1 vez; cinco6 vezes, sendo tres e dois - 3 vezes. quatro um - 1 vez, quatro - 4 vezes, sendo um e tres - 2 vezes e dois e dois - 2 vezes; tres - 2 vezes sendo dois $e$ um -2 vezes; dois - 1 vez, sendo un e un - 1 vez; sete - 1 vez, sendo quatro e tres - 1 vez. Em outras 8 vezes $(26,68 \pm 8,1)$ a iprigacro da porcyo cervical do timo depende tambem de colaterais oriundos de ambas as Aa. carotidas comuns todavia associados, sempre, de un ramo procedente da dea cranial esquerda. 5 vezes $(16,6 \% t$ 6,8), da A. toracica externa esquerda, 2 vezes $(6,6 \% \pm 4,5)$, da $A$. torbcica interna esquerda, 1 vez $(3,3 \% \pm 3,3)$. Nestes casos as Aa carotidas comuns direita e esquerda cedem, tambem, numero variavel de contribuicores que, considerado igualmente, por ordem, o numero total e o seu lado de origen, mostra a seguinte distribuicso: quatro -2 vezes, sendo um e dois -2 vezes; seis - 2 vezes, sendo dois e tres. 2 vezes; sete - 2 vezes, sendo tres e tres - 1 vez e quatro dois - 1 vez; oito - uma vez, sendo quatro e tres - 1 vez; nove - 1 vez, sendo quatro e quatro - 1 vez.

Apenas 1 vez $(3,3 x+3,3)$ o suprimento sanguineo da porca cervical do timo e estabelecido por tres colaterais da $A$. carotida comum ojreita, aos quais somam-se, nesta tarefa, uma ramo procedente da $R$. tiroidea cranial esquerda e outro da $A$. toracica externa esquerda.

Cabe salientar que os colaterais emitidos pelas Aa. carotidas comuns direita esquerda, responsaveis pela irrigasso da porcaro cervical do t 1 mo, mais comumente, procedem de colaterais que tambem emitem ramos destinados is estruturas vizintas, principalmente a musculatura da regiao, ou como en. alguns casos, atingem somente 0 !́mo, sendo os primeiros tidos como vasos
Indiretos (CEm e [Dm) e os ultimos como diretos (CE e CD), conferindo caracterlsticas proprias, a estes diferentes arranjos vasculares - Duad. 1 ).

QUADRO 1 - Namero de vasos originados direta ( $C E$ e CD) ou indiretamente (CEm e $[D m$ ) das Pa. carotidas comuns esquerda e direita, quando syo responstreis pela ir rigacgo total da porcgo cervical do timo lobs. 2, 4, 6, 7, 8, 9, $10,13,14,15,16,18,19,20$, $22,23,25,26,28,29,30$ ), ou quando se associa uma contribuicro oriunda da $A$. tiroidea cranial esquerda CObs. 1, 17, 17, 21, 27), da A. toracica externa esquerda (Obs. 12, 24), da $A$. toracica interna esquerda (Obs. 3) $e$ da $A$. toracica externa esquerda juntamente com a $A$. tiroidea cranial esquerda cobs. 5). SP, 1984 .

\begin{tabular}{|c|c|c|c|c|c|}
\hline 085. & & CE & CEm & $C D$ & $\mathrm{CDm}$ \\
\hline 1 & 1 & 1 & $=7$ & 7 & \\
\hline 2 & 1 & 2 & 0 & 2 & 1 \\
\hline 3 & 1 & 0 & 2 & 0 & 1 \\
\hline 4 & i & 1 & 0 & 0 & 2 \\
\hline 5 & 1 & 0 & 0 & 1 & 2 \\
\hline 6 & 1 & 0 & 1 & 2 & 2 \\
\hline 7 & 1 & 1 & 2 & 0 & 1 \\
\hline 8 & I & 1 & 1 & 2 & 1 \\
\hline 9 & I & 0 & 1 & 0 & 1 \\
\hline 10 & 1 & 0 & 3 & 0 & 3 \\
\hline 11 & 1 & 1 & 2 & 1 & 2 \\
\hline 12 & I & 0 & 4 & 1 & 3 \\
\hline 13 & I & 1 & 2 & 1 & 3 \\
\hline 14 & I & 0 & 2 & 0 & 2 \\
\hline 15 & I & 0 & 1 & 0 & 2 \\
\hline 16 & 1 & 0 & 2 & 0 & 2 \\
\hline 17 & I & 1 & 2 & 0 & 2 \\
\hline $1 B$ & I & 0 & 2 & 0 & 3 \\
\hline 19 & I & 0 & 2 & 1 & 3 \\
\hline 20 & I & 1 & 2 & 0 & 1 \\
\hline 21 & I & 0 & 2 & 0 & 4 \\
\hline 22 & 1 & 0 & 4 & 0 & 2 \\
\hline 23 & I & 0 & 3 & 1 & 2 \\
\hline 24 & 1 & 2 & 1 & 0 & 2 \\
\hline 25 & 1 & 2 & 1 & 0 & 2 \\
\hline 26 & I & 1 & 3 & 0 & 2 \\
\hline 27 & I & 1 & 1 & 0 & 1 \\
\hline 28 & I & 0 & 3 & 0 & 2 \\
\hline 29 & I & 0 & 3 & 1 & 2 \\
\hline 30 & I & 0 & 3 & 0 & 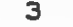 \\
\hline
\end{tabular}

ด andise estatlstica dos resultados mostra-nos que a participacro dos diferentes vasos encontrados como responsabeis pela nutricgo do tlmn. nos bovinos de origem europeia, independente do sexu.

Finalmente, considerendo conjuntamente - numero, procedencia e ordenaça de distribuiço das arterias destinadas as porcotes torbcicas e cervical do $\$ 1$ mo, podemos identificar diferentes modalidades da vascularizaço, que registramos nos auad. 2 e 3.

Rev.Fac.Med.vet.Zootec.Univ.S.Paulo, 23(1):31-38. 1986. 


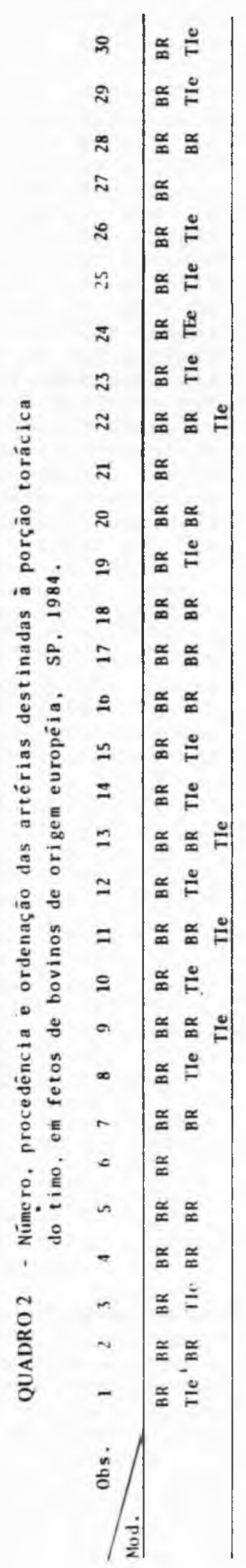

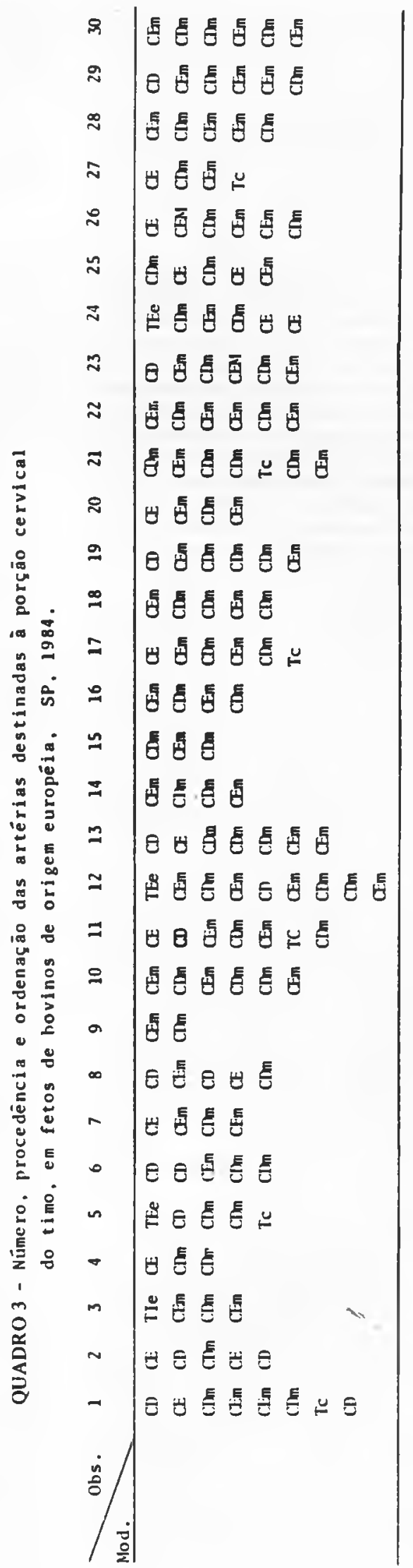




\section{COMENTARIOS E CONCLUSDES}

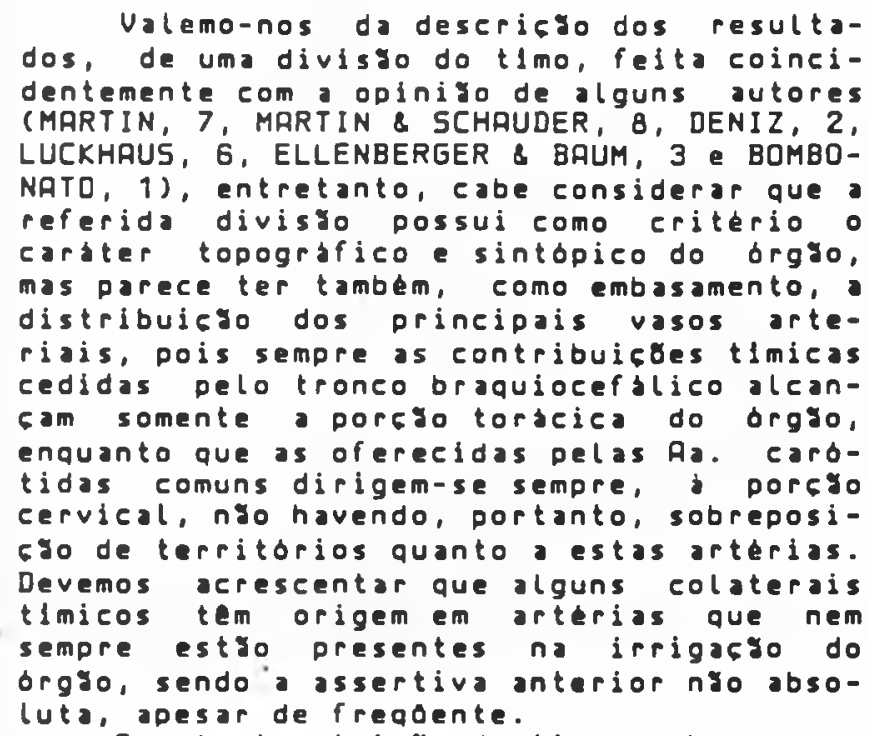

Quanto butricto do tlmo, observanos achados semelhantes as descricoles genericas fejtas pelos tratadistas que, apesar de no fazerem, mencyo as contribuicóses cedidas pela $A$ a. torbcica externa esquerda e tiroidea cranial esquerda, relatam aquelas oriundas da A. subelsuia, fato por nos observado. Ja, confrontando a literatura especializada, notamos que DENIZ, 2, faz mencyo a contribuicyo procedente da $A$. vertebralis, enquanto que LUCKHAUS, 6, eita a participacyo de colaterais do ramus descendens da A. oceipitalis e R. palatina ascendens por nos nao encontrados. Em relacro descricjo de BOMBONATO, 1, evidenciamos como diferenca a participacro da $A$. torbcica externa apenas nos taurinos e do tronco omocervical somente nos azebuadas. como tambem diferencas no namero nais freqoente de vasos.

DENIZ, 2, salienta que o nomero de vasos timicos depende do tamanho do feto: podemos, assim, admitir tendencia semelhante nos nossos resultados, como sugerem os Fig. 1 e 2 . Todavia, afirmacro conclusi$v$ deste importante fato depende ainda, a nosso ver. de estudos a serem efetuados em amostragens diferentes e en outras especies, bem cono do melhor conhecimento da involucro deste orgyo.

Finalmente, andise dos resultados permite-nos conclulr:

- tlmo recebe contribuicoes procedentes do tronco braquiocefalico, das Aa. carotidas comuns direjta e esquerda. da A. toracica interna esquerda, da a.

\begin{abstract}
tordcica externa e da a.tireoidea cranial esquerda. Independentemente das suas origens, os colaterais timicos estyo presentes en numero de: ol to - 8 vezes $(26,78 \pm 8,1)$, sete -7 vezes $(23,48 \pm 7,7)$, seis - 6 vezes $(20,08 \pm 7,3)$, cinco -4 vezes $(13,38$ $\pm 6,2)$, dez - 3 vezes $(10,08 \pm 5,5)$, nove - 1 vez $(3,35 \pm 3,3)$, onze - 1 vez $(3,38 \pm 3,3)$.

no concernente anslise conjunta do numero, procedencia e ordenaço das arterias, observamos possuir cada preparacio arranjo vascular proprios

ngo foram reveladas diferencas estat1sticamente significantes $(=5,0 \$)$ no tocante ao arranjo vascular, quando testamos o sexo.
\end{abstract}

Esquemas do timo de fetos de bovinos de orlgen europeia, mostrando o menor (Flg.3), - maior (Fig. 4) e o mais freqôente (Fig.5) idmero de ramos arteriais responsaveis pela lrrigacyo do orgaso.

BOMBONATO, P.P. , BORELLI, U., FERNANDES FILHO, A. Contribution to the study of arteriat vascularization in bovine Foetuses cBos taurusi. Rev. Foc. Hed.vet.zootec. Undv.S.Psulo, $23(1): 31-38,1986$.

SUMmaRY: The orlgin, number and ordering of the inymics arteries were studied in 30 bovine foetuses (Bos taurus), 16 remole and 14 male in vaplous stages of development. They had been infected with solution of Neoprene latex $1450^{\circ}$ and fixed in formol ot 10.08 . The thymus recives contribution from the brachiocephalic trunk. and from the right and the left common carotid ortarles. from the left internal thoracic ortery. from the left external thoracic artery and from the left crantal thyroid ortery. It yas observed several thymics collaterals that is elght - 8 cases; seven - 7 casesi six - 6 casess five - 4 cases; ten - 3 cases: nine - 1 case; eleven - 1 case. Finally, concerning the aspects of ordering, it is own of each case. UNITERMS: Anatomy of catle: $i^{+}$Arteriesi $i^{+}$Thymus 


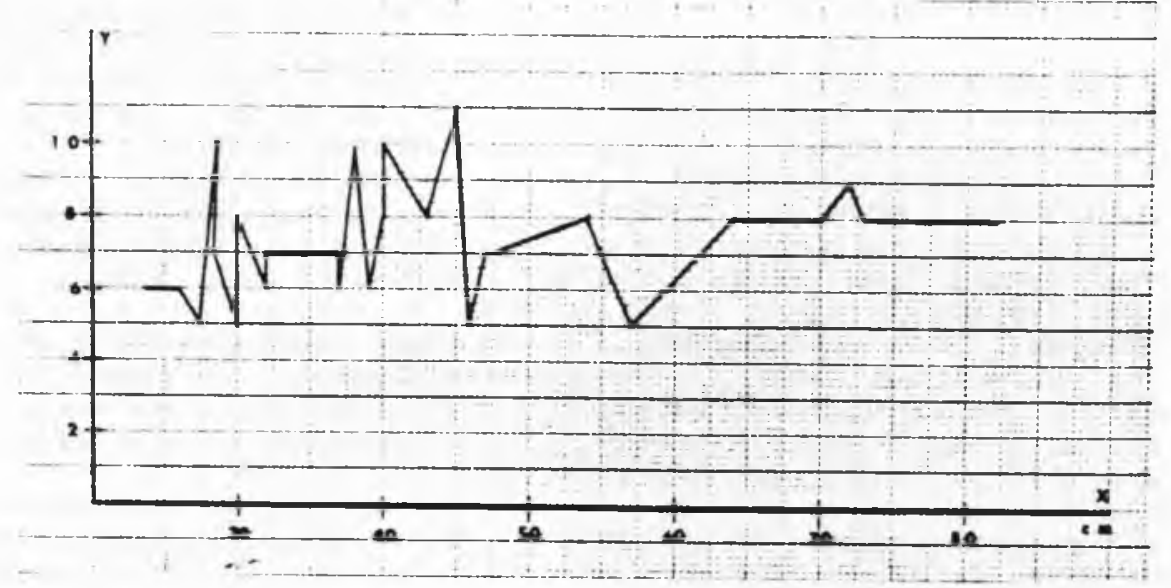

FIGURA 1 - Gráfico representativo da distribuiçāo do número total de artérias destinadas ao timo $(y)$ em relaçāo ao comprimento do feto $(\mathbf{x})$.

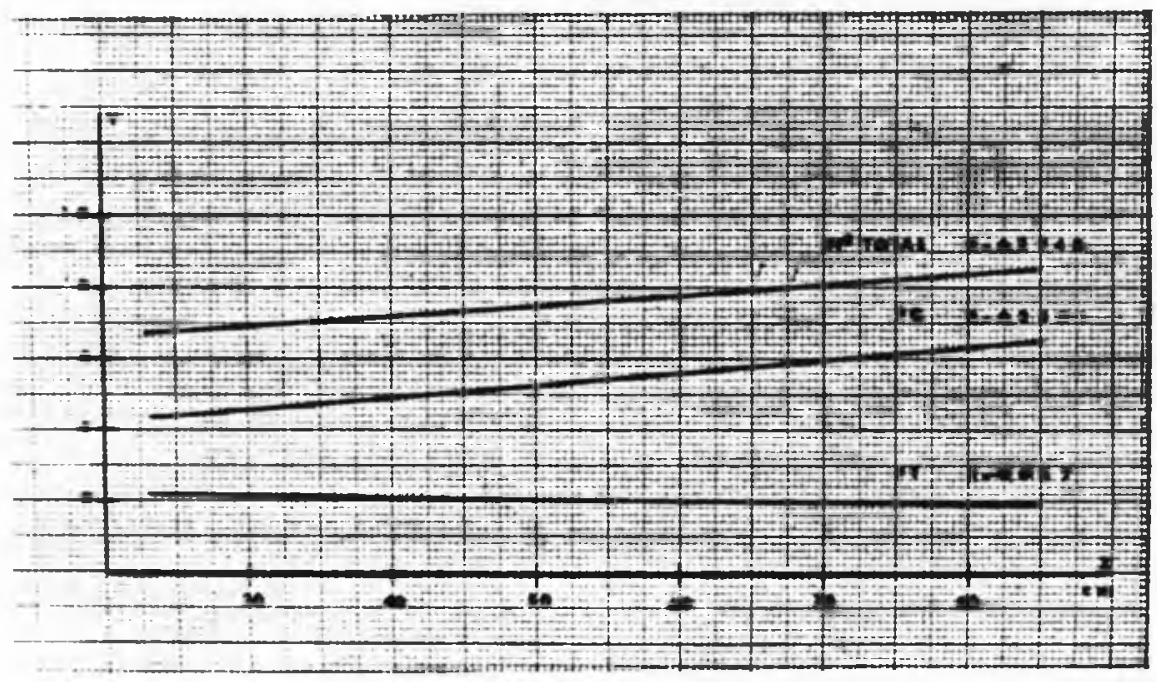

FIGURA 2 - Gráfico representativo da regressão Linear da distribuiçāo do número de artérias destinadas ao timo $(y)$, em relação ao comprimento do feto $(\mathrm{x})$. 

origem européia (Bos tourus).

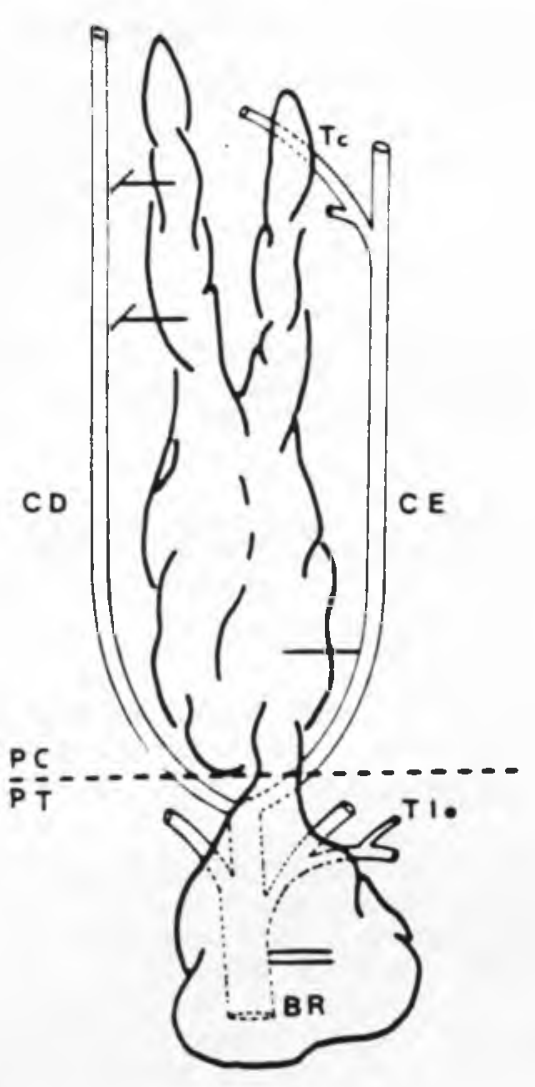

FIGURA 3

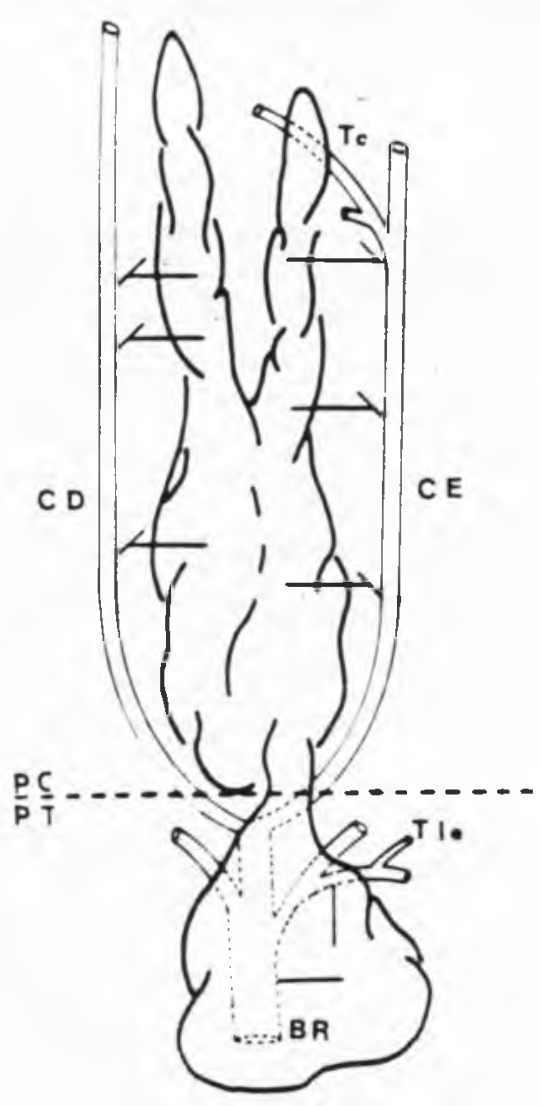

FIGURA 5

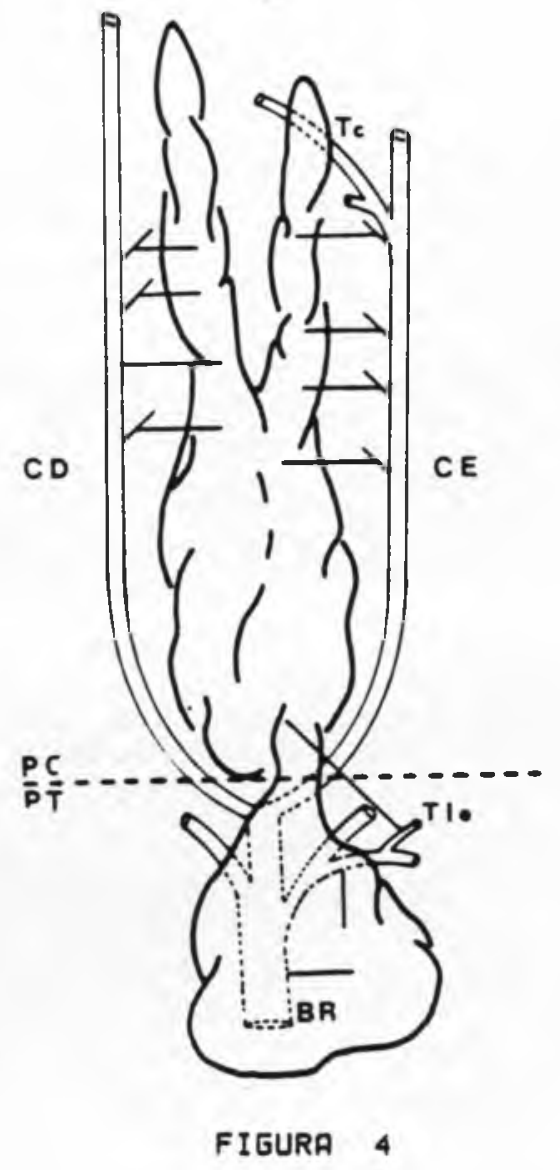

\section{LEGENDA}

BR-Tronco braquiocefalico.

CD-A. carbtida comum direita.

CE-A. carotida comum esquerda.

PC-Porcto cervical.

PT-Porero toracica.

Te-R. tirojdea cranial esquerda.

TIe-p. torbcica interna esquerda. 
REFERENCIAS BIBLIOGRAFICAS

1 - Bomgonato, P.P. Contribuiço vo estudo da vascularizacro arterial do timo en fetos de bovinos azebuados. 5yo Paulo, 1982. 57p. lTese de mestrado - Faculdade de Medicina Veterinaria e Zootecnia da Universidade de 5ro Paulol.

2 - DENIZ, E. Die Blutgef abversorgung des Thymus bein Kalb. Zbl. Vet. Med.. 11-A:750-8, 1964 .

4 - GETTY, R. Sisson and Grossman's, the anatomy of the domestic animals. 5.ed. Philadelphia, W.B. Saunders, 1975. $\times .1$, p.1064.

3 - ellengerger, w. \& Baum, H. Handbuch der vergleichenden Anatomie der Haustiere. $18.0 d$. Berlim, J. Springer. 1977. D.802-604.

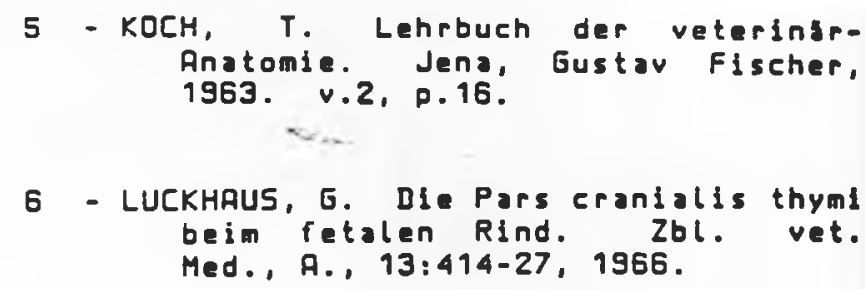

7 - MARTIN, P. Lehrbuch der Anatomie der Haustiere. Stuttgard, Schickhardt \& Ebner, 1902. v.1, p.430.

8 - MARTIN, P. \& SCHAUDER, N. Lehrbuch der Anatomie der Haustiere. Stuttgard, Schickhardt \& Ebner, 1938. v.3, p.370.

9 - OLIVEIRA, M.C.; ORSI, A.M.; PINTO E SILUA, P.; MELLO DIAS, A. Sobre a remescencia da arteria tiroidea cranial em bovinos (Bos indicus). Cientifica, Jaboticabal, $6 \mathrm{ln}$. especial):55-7, 1979 .

Recebldo para publicacyo en $26 / 06 / 85$
Aprovado para publicacyo em $09 / 04 / 86$ Inpresso en $19 / 86$ 